Research Article

\title{
Association of rs944289, rs965513, and rs1443434 in TITF1/TITF2 with Risks of Papillary Thyroid Carcinoma and with Nodular Goiter in Northern Chinese Han Populations
}

\author{
Xin Zhang $\mathbb{D}^{1,}, 2$ Yulu Gu $\left(\mathbb{D},{ }^{1}\right.$ Yong Li $\left(\mathbb{D},{ }^{1}\right.$ Heran Cui ${ }^{1 D},{ }^{1}$ Xiaoli Liu, ${ }^{3}$ Hui Sun $\left(\mathbb{D},{ }^{3}\right.$ \\ Qiong Yu $\left(\mathbb{1},{ }^{1}\right.$ Yaqin Yu $\left(\mathbb{D},{ }^{1}\right.$ Yawen Liu $\mathbb{1}$, ${ }^{1}$ Siyan Zhan $\mathbb{D}^{1,4}$ and Yi Cheng $\mathbb{D}^{5}$ \\ ${ }^{1}$ Department of Epidemiology and Biostatistics, School of Public Health, Jilin University, Changchun 130021, China \\ ${ }^{2}$ Department of Pharmacy, The First Hospital of Jilin University, Changchun 130021, China \\ ${ }^{3}$ Jilin Provincial Key Laboratory of Surgical Translational Medicine, Department of Thyroid and Parathyroid Surgery, \\ China-Japan Union Hospital, Jilin University, Changchun 130033, China \\ ${ }^{4}$ Department of Epidemiology and Biostatistics, School of Public Health, Peking University Health Science Centre, \\ Beijing 100191, China \\ ${ }^{5}$ Department of Cardiovascular Center, First Hospital of Jilin University, Changchun 130021, China
}

Correspondence should be addressed to Siyan Zhan; siyan-zhan@bjmu.edu.cn and Yi Cheng; chengyi@jlu.edu.cn

Received 16 November 2019; Revised 25 December 2019; Accepted 30 December 2019; Published 11 February 2020

Academic Editor: Maria L. Dufau

Copyright (c) 2020 Xin Zhang et al. This is an open access article distributed under the Creative Commons Attribution License, which permits unrestricted use, distribution, and reproduction in any medium, provided the original work is properly cited.

Objective. In this study, we aimed to investigate the associations of three single-nucleotide polymorphisms (SNPs) on TITF1/ TITF2 (rs944289, rs965513, and rs1443434) with susceptibility to papillary thyroid carcinoma (PTC) and with nodular goiter (NG) in northern Chinese Han populations. Methods. We performed a case-control study comprising 861 PTC patients, 562 NG patients, and 896 normal controls (NCs). One TITF1 SNP (rs944289) and two TITF2 SNPs (rs965513 and rs1443434) were genotyped. Departures from Hardy-Weinberg equilibrium (HWE) in the control group were evaluated using chi-square test. Associations of the SNPs with PTC and with NG were assessed by unconditional logistic regression using the online SNPStats program. Bonferroni correction was performed for multiple tests in genotype analyses. Data analysis was performed by SPSS24.0 unless otherwise specified. Results. For rs944289, T allele was associated with increased risks for both PTC (OR $=1.23,95 \%$ CI: $1.08-1.41, P=0.002)$ and NG $(\mathrm{OR}=1.28,95 \% \mathrm{CI}: 1.10-1.50, P=0.002)$, and TT genotype significantly increased NG risk (recessive model, $\mathrm{OR}=1.60,95 \% \mathrm{CI}: 1.22-2.10, P=0.001$ ). For rs965513, no association was observed after Bonferroni correction. For rs1443434, G allele was associated with increased PTC risk $(\mathrm{OR}=1.33,95 \% \mathrm{CI}$ : $1.10-1.61, P=0.003)$. Moreover, PTC risk increased with the number of total risk alleles of the three SNPs $(\mathrm{OR}=1.25,95 \%$ CI: $1.13-1.37, P<0.001)$. After stratified by gender, the risk effect of rs944289 T allele on PTC was only observed in females (OR=1.29, 95\% CI: $1.10-1.50, P=0.001)$. Individuals carrying rs944289-rs965513-rs 1443434 haplotypes T-G-G and T-G-T had increased risks of PTC (OR $=1.82,95 \%$ CI: $1.25-2.64, P=0.002)$ and NG $(\mathrm{OR}=1.28,95 \% \mathrm{CI}: 1.06-1.54, P=0.011)$, respectively. Conclusions. There are associations of rs944289 and rs1443434 polymorphisms with PTC risk and association of rs944289 polymorphism with NG risk. Haplotypes T-G-G and T-G-T are risk haplotypes of PTC and NG, respectively.

\section{Introduction}

Thyroid cancer, as one of the most common tumor of endocrine system, is the fifth leading cancer for the estimated new cancer cases in females $[1,2]$. Among all the histological subtypes of thyroid cancer, papillary thyroid carcinoma
(PTC) is the most common type, accounting for about $75-85 \%$ of all cases with thyroid cancer.

Genetic factors are essential for the pathogenesis of thyroid cancer [3]. Thyroid cancer has the highest heritability (53\%) among 15 common cancers, with very low shared environmental effects (1\%) [4]. A joint study from 
five Nordic countries revealed that lifetime cumulative risk of female relatives of PTC patients $(2 \%)$ is higher than that of males (1\%); the more PTC patients a family has, the more risk of thyroid cancer their relatives has; and cumulative risk of thyroid cancer increases threefold in comparison with general population lifetime [5]. Moreover, a study from the Korea National Health and Nutrition Examination Surveys showed that factors related to thyroid function, such as thyroid-stimulating hormone (TSH) and free thyroxine (fT4), also present high heritability: the narrow-sense heritability for TSH is $54 \%$, and that for free thyroxine (fT4) is $56 \%$; the narrow-sense heritability is higher in females than that in males for TSH; and both TSH and fT4 show negative genetic correlations in females after adjusted for environmental effects [6]. In a twin study including 69 monozygotic and 45 dizygotic adult twin pairs, the environment-adjusted heritability of the thickness of thyroid isthmus and thyroid volume (left and right lobes) on thyroid cancer was 50\% and $68-79 \%$, respectively [7]. These evidences reflect the high heritability of thyroid cancer.

Molecular mechanisms (gene mutation, metastasis suppressors abnormally expression, epigenetic modification, microRNA regulation, and signaling pathway alteration) are involved in thyroid cancer pathogenesis [8, 9]. The $\mathrm{BRAF}^{\mathrm{V} 600 \mathrm{E}}$ mutation and RAS mutation has been identified as the most prevalent oncogenic event in thyroid cancer [9]. Novel risk loci on different genes have further been identified and validated in different populations, providing a new dimension to this extensive line of thyroid cancer research $[10,11]$. TITF1 and TITF2 are located on chromosome $14 q 13.3$ and $9 q 22.33$, respectively, encoding thyroid-specific transcription factors (TITF1 and TITF2) $[12,13]$. TITF1 is a homeodomain-containing nuclear transcription factor, being crucial in thyroid development and differentiation by regulating thyroid-specific genes thyroglobulin ( $\mathrm{Tg}$ ), thyroperoxidase (TPO), and thyrotropin receptor (TSHR) [14]. TITF2 contains a forkhead domain and encodes a phosphorylated protein. Mutations in TITF2 lead to the Bamforth syndrome, penetrating as thyroid agenesis [15]. These indicate that important loci mutations on TITF1 and TITF2 may modify the expression of these two transcription factors and influence their function on thyroid gland. Recently, different single-nucleotide polymorphisms (SNPs) in TITF1 (also called NKX2-1 and TTF1)/TITF2 (also called FOXE1 and TTF2) are found in PTC, suggesting that rs944289, rs965513, and rs1443434 may be implicated in PTC risk $[2,13]$. However, these studies are performed in populations with different ethnic background, conferring inconsistent results [16-18].

In this study, we evaluated the association of rs944289, rs965513, and rs1443434 in TITF1/TITF2 with PTC risks and with nodular goiter (NG) in northern Chinese Han populations.

\section{Materials and Methods}

2.1. Patients and Samples. A total of 861 patients with PTC, 562 patients with NG, and 896 normal controls (NCs) were included in this study. The patients with PTC and the patients with NG were recruited from January 2012 to December 2014 in the China-Japan Union Hospital of Jilin University, China. The inclusion criteria of PTC and NG patients included the followings: (1) northern Chinese Han populations; (2) pathologically diagnosed based on the Revised American Thyroid Association Management Guidelines for patients with thyroid nodules and differentiated thyroid cancer [19]; (3) histologic type of PTC was pure papillary carcinoma other than follicular variant of PTC (fvPTC). The NCs were selected from a large-scale community-based cross-sectional survey of chronic disease and risk factors among adults in Jilin province conducted in July 2012. All the NCs were northern Chinese Han populations in community with the following exclusion criteria: (1) with metabolic disease (hypertension, diabetes, and dyslipidemia), (2) with any thyroid disease (thyroiditis, benign and malignant thyroid tumors), (3) with other malignancy, and (4) with mental disorders. Demographic and clinical information of the patients and controls were obtained through inquiry or consulting medical records. Peripheral blood was provided by each participant for genotyping. The study was approved by the ethics committee of the School of Public Health, Jilin University, and written informed consents were obtained from the patients and controls in the study.

2.2. Selection of SNPs. We selected rs944289 in TITF1, and rs965513 and rs1443434 in TITF2 in this study based on previous genome-wide association studies (GWAS) and confirmatory studies $[18,20-22]$.

2.3. DNA Extraction and SNP Genotyping. Genomic DNA extraction and SNP genotyping were performed as in our previously published study [23]. Briefly, $5 \mathrm{ml}$ of peripheral blood obtained from each patient and control was stored in EDTA-containing tubes. Genomic DNA was extracted using a commercial DNA extraction kit (ClotBlood DNA kit, Cwbio, Beijing, China) according to the manufacturer's instructions. Primers for polymerase chain reaction (PCR) were designed by Assay Designer 3.1 (Supplementary Table S1). Genotypes were detected by matrix-assisted laser desorption/ionization time of flight mass spectrometry (MALDI-TOF-MS) using the MassARRAY system (Sequenom, San, Diego, CA, USA). The genotyping rate of rs944289, rs965513, and rs 1443434 was $99.09 \%, 97.15 \%$, and 95.34, respectively.

2.4. Statistical Analysis. Continuous variables were presented with mean and standard deviation, and categorical variables were presented with percentage. Differences between groups were tested on the basis of independentsamples $t$-test or chi-square test using SPSS Statistics 24.0 (IBM Canada Ltd.). Departure from Hardy-Weinberg equilibrium (HWE) in NCs was calculated on the basis of chi-square test using SPSS Statistics 24.0 (IBM Canada Ltd.). Associations between SNPs and PTC/NG under five models of inheritance (codominant, dominant, recessive, 
overdominant, and allele model) were performed using online SNPStats program (http://bioinfo.iconcologia.net/ SNPStats) [24]. Strength of association was assessed by odds ratio (OR) and corresponding 95\% confidence intervals (CIs). The best model of inheritance for each SNP was selected on the basis of the Akaike information criterion (AIC). Bonferroni correction was performed to reduce type I error in multiple testing in view of 15 comparisons between groups (three SNPs $\times$ five models); thus, significant $P$ value was set at $0.05 / 15=0.003$ for genotype analyses. Linkage disequilibrium analysis between the three SNPs and haplotype analysis was analyzed using the online SNPStats program. Power calculations were done using Quanto 1.2.4 software. Two-sided test with $P$ value less than 0.05 was considered statistically significant otherwise specified.

\section{Results}

3.1. Characteristics of the Study Subjects. The age and gender distribution were shown in Table 1. Significant difference of age distribution was observed between NG and NC $(P<0.05)$. The female-to-male ratio was more than 3.0 in the three groups, and no significant difference of gender distribution was found among the three groups $\left(\chi^{2}=0.008\right.$, $P=0.996)$. Clinical characteristics of PTC were shown in Supplementary Table S2. In this study, 15.7\% of the patients with PTC had a family history; $34.7 \%$ of the patients with PTC were at stage 0-I; and $18.4 \%$ of the PTCs had lymph node metastasis.

3.2. Genotype and Allele Distributions of the Three SNPs. Significant differences of genotype and allele distributions among the groups were found for all the three SNPs (all $P<0.05$ ) (Table 2). For genotype distribution among the groups, chi-square test of linear-by-linear association was also performed, and significant trend associations were found for all the three SNPs $\left(P_{\text {trend }}<0.05\right)$. No departure from HWE was observed in NC group (for rs944289, $P=0.669$; for rs965513, $P=0.127$; and for rs1443434, $P=0.974)$. Linkage disequilibrium between the three SNPs was analyzed using the online SNPStats program, and no significant linkage disequilibrium was found $(P>0.05)$ (Supplementary Table S3). To make our results more dependable and avoid false-positive results, we also analyzed the difference of genotype and allele distributions with 1000 genome Han Chinese in Beijing (CHB) as controls, and the results were shown in Supplementary Table S4. Except for rs1443434 $\left(P_{\text {trend }}=0.012\right)$, no significant distribution difference was found for rs944289 and rs965513 $(P>0.05)$.

3.3. Association between the Three SNPs and PTC/NG. We used codominant, dominant, recessive, overdominant, and allele models to evaluate associations of rs944289, rs965513, and rs1443434 polymorphisms with PTC and with NG (Tables 3-5). Because of the significant age difference between NG and NC, evaluation of associations between SNPs and NG was adjusted by age. Codominant model was detected as the best model for all the three SNPs in the analysis of associations between the SNPs and PTC risk, and recessive model was detected as the best model for all the three SNPs in the analysis of association between the SNPs and NG risk. For rs944289, T allele was associated with increased risks for both PTC (OR $=1.23,95 \%$ CI: 1.08-1.41, $P=0.002)$ and $\mathrm{NG}(\mathrm{OR}=1.28,95 \% \mathrm{CI}: 1.10-1.50$, $P=0.002)$. TT genotype significantly increased PTC risk (codominant model, $\mathrm{OR}=1.52, \quad 95 \%$ CI: $1.15-1.99$, $P=0.010$ ) and NG risk (recessive model, $\mathrm{OR}=1.60,95 \% \mathrm{CI}$ : $1.22-2.10, P=0.001$ ); however, the risk effect of TT genotype on PTC was not significant after Bonferroni correction. For rs965513, A allele was associated with increased PTC risk $(\mathrm{OR}=1.34,95 \% \mathrm{CI}: 1.08-1.67, P=0.007)$, and AA genotype significantly increased PTC risk under codominant model $(\mathrm{OR}=3.81,95 \% \mathrm{CI}: 1.25-11.63, P=0.008)$. NG risk increased significantly for AA genotype under recessive model $(\mathrm{OR}=4.53,95 \%$ CI: $1.41-14.59, P=0.006)$. However, after Bonferroni correction, no association was still significant for rs965513. For rs1443434, G allele was associated with increased PTC risk (OR $=1.33$, 95\% CI: $1.10-1.61, P=0.003)$. Under the codominant model, GG genotype of rs1443434 significantly increased PTC risk $(\mathrm{OR}=2.21,95 \%$ CI: $1.15-4.23, P=0.010$ ), but the association was not significant after Bonferroni correction. Powers were calculated under dominant and recessive models using Quanto 1.2.4 software on the basis of the sample size and minor allele frequencies in our study (Supplementary Table S5). We also performed the association analyses with 1000 genome $\mathrm{CHB}$ as controls (Supplementary Table S6-S8). No genotype or allele on the three SNPs was found significantly associated with PTC $(P>0.05)$. For rs1443434, $\mathrm{G}$ allele was associated with increased risk of NG (OR $=1.87,95 \%$ CI: $1.05-3.31, P=0.04)$, but the association was not significant after Bonferroni correction.

3.4. Cumulative Risk Effect of the Three SNPs on PTC. We performed chi-square test of linear-by-linear association and logistic regression to evaluate the combined effect of the three SNPs on PTC risk (Table 6). Total counts of risk alleles for each subject were calculated, with a range of $0-5$. As a result, percentages of PTCs with more risk alleles (three, four, and five) were higher than that of NGs and NCs $\left(P_{\text {trend }}<0.001\right)$, and with the increasing of each risk allele, PTC risk increased 0.25 -fold (OR $=1.25,95 \%$ CI: 1.13-1.37, $P<0.001)$.

3.5. Haplotype Analysis. Haplotype analysis revealed that individuals carrying rs944289-rs965513-rs1443434 haplotypes T-G-G and T-G-T had increased risks of PTC (OR $=1.82,95 \%$ CI: $1.25-2.64, P=0.002)$ and of NG $(\mathrm{OR}=1.28,95 \%$ CI: 1.06-1.54, $P=0.011$ ) (Supplementary Table S9-S10), respectively.

3.6. Association between the Three SNPs and PTC Risks after Stratified by Gender. Genotype and allele frequencies of the three SNPs in females and males are shown in Supplementary Table S11. After stratified by gender, associations 
TABle 1: The age and gender distribution between PTCs, NGs, and NCs $[n(\%)]$.

\begin{tabular}{lcccr}
\hline Gender/age & PTC & NG & NC & $\chi^{2} / F$ \\
Age & $43.7 \pm 9.2$ & $48.5 \pm 10.0^{*}$ & $43.7 \pm 9.1$ & 56.299 \\
Gender & & & & \\
$\quad$ Male & $210(24.4)$ & $138(24.6)$ & $220(24.6)$ & 0.008 \\
$\quad$ Female & $651(75.6)$ & $423(75.4)$ & $676(75.4)$ & 0.996 \\
\hline
\end{tabular}

PTC, papillary thyroid carcinoma; NG, nodular goiter; NC, normal control; ${ }^{*}$ NG vs. NC, $P<0.05$.

TAвLE 2: Genotype and allele distributions of the three SNPs in PTCs, NGs, and NCs.

\begin{tabular}{|c|c|c|c|c|c|c|}
\hline Genotype/allele & PTC & NG & $\mathrm{NC}$ & $\chi^{2}$ & $P$ & $P_{\text {trend }}$ \\
\hline \multicolumn{7}{|l|}{ rs944289 } \\
\hline $\mathrm{C} / \mathrm{C}$ & $255(29.8)$ & $176(31.7)$ & $315(35.5)$ & 16.473 & 0.002 & 0.003 \\
\hline $\mathrm{C} / \mathrm{T}$ & $416(48.7)$ & $244(43.9)$ & $422(47.6)$ & & & \\
\hline $\mathrm{T} / \mathrm{T}$ & $184(21.5)$ & $136(24.5)$ & $150(16.9)$ & & & \\
\hline $\mathrm{C}$ & $926(54.2)$ & $596(53.6)$ & $1052(59.3)$ & 12.821 & 0.002 & \\
\hline $\mathrm{T}$ & $784(45.8)$ & $516(46.4)$ & $722(40.7)$ & & & \\
\hline \multicolumn{7}{|l|}{ rs965513 } \\
\hline $\mathrm{G} / \mathrm{G}$ & $647(76.8)$ & $449(82.2)$ & 704 (81.4) & 16.768 & 0.002 & 0.007 \\
\hline $\mathrm{A} / \mathrm{G}$ & $181(21.5)$ & $85(15.6)$ & $157(18.2)$ & & & \\
\hline $\mathrm{A} / \mathrm{A}$ & $14(1.7)$ & $12(2.2)$ & $4(0.5)$ & & & \\
\hline G & $1475(87.6)$ & $983(90.0)$ & $1565(90.5)$ & 8.181 & 0.017 & \\
\hline $\mathrm{A}$ & $209(12.4)$ & $109(10.0)$ & $165(9.5)$ & & & \\
\hline \multicolumn{7}{|l|}{ rs1443434 } \\
\hline $\mathrm{T} / \mathrm{T}$ & $590(70.7)$ & 409 (78.5) & $651(76.1)$ & 18.014 & 0.001 & 0.003 \\
\hline $\mathrm{T} / \mathrm{G}$ & $217(26.0)$ & $107(20.5)$ & $190(22.2)$ & & & \\
\hline $\mathrm{G} / \mathrm{G}$ & $28(3.4)$ & $5(1.0)$ & $14(1.6)$ & & & \\
\hline $\mathrm{T}$ & $1397(83.7)$ & $925(88.8)$ & $1492(87.3)$ & 16.533 & $<0.001$ & \\
\hline G & $273(16.3)$ & $117(11.2)$ & $218(12.7)$ & & & \\
\hline
\end{tabular}

$P_{\text {trend }}, P$ value of linear-by-linear association test. PTC, papillary thyroid carcinoma; NG, nodular goiter; NC, normal control. Significant $P$ values in bold.

TABLE 3: Association between rs944289 polymorphism and PTC/NG.

\begin{tabular}{|c|c|c|c|c|c|c|}
\hline \multirow{2}{*}{ Genotype/allele } & \multicolumn{3}{|c|}{ PTC vs. NC } & \multicolumn{3}{|c|}{ NG vs. NC } \\
\hline & OR $(95 \% \mathrm{CI})$ & $P$ & AIC & OR $(95 \% \mathrm{CI})^{*}$ & $P^{*}$ & AIC \\
\hline \multicolumn{7}{|l|}{ Codominant } \\
\hline $\mathrm{C} / \mathrm{C}$ & 1.00 & 0.010 & 2411.1 & 1.00 & 0.003 & 1835.4 \\
\hline $\mathrm{C} / \mathrm{T}$ & $1.22(0.98-1.51)$ & & & $1.06(0.83-1.36)$ & & \\
\hline $\mathrm{T} / \mathrm{T}$ & $1.52(1.15-1.99)$ & & & $1.66(1.22-2.26)$ & & \\
\hline \multicolumn{7}{|l|}{ Dominant } \\
\hline $\mathrm{C} / \mathrm{C}$ & 1.00 & 0.011 & 2411.9 & 1.00 & 0.092 & 1842.5 \\
\hline $\mathrm{C} / \mathrm{T}+\mathrm{T} / \mathrm{T}$ & $1.30(1.06-1.58)$ & & & $1.22(0.97-1.54)$ & & \\
\hline \multicolumn{7}{|l|}{ Recessive } \\
\hline $\mathrm{C} / \mathrm{C}+\mathrm{C} / \mathrm{T}$ & 1.00 & 0.014 & 2412.4 & 1.00 & 0.001 & 1833.6 \\
\hline $\mathrm{T} / \mathrm{T}$ & $1.35(1.06-1.71)$ & & & $1.60(1.22-2.10)$ & & \\
\hline \multicolumn{7}{|l|}{ Overdominant } \\
\hline $\mathrm{C} / \mathrm{C}+\mathrm{T} / \mathrm{T}$ & 1.00 & 0.650 & 2418.1 & 1.00 & 0.240 & 1844.0 \\
\hline $\mathrm{C} / \mathrm{T}$ & $1.04(0.87-1.26)$ & & & $0.88(0.70-1.09)$ & & \\
\hline \multicolumn{7}{|l|}{ Allele } \\
\hline C & 1.00 & 0.002 & - & 1.00 & 0.002 & - \\
\hline $\mathrm{T}$ & $1.23(1.08-1.41)$ & & & $1.28(1.10-1.50)$ & & \\
\hline
\end{tabular}

${ }^{*}$ Adjusted by age. PTC, papillary thyroid carcinoma; NG, nodular goiter; NC, normal control. Bonferroni correction was performed, and $P<0.003$ was defined as statistical significance. Significant $P$ values after Bonferroni correction in bold.

between the three SNPs and PTC risk under codominant, dominant, recessive, overdominant, and allele models are shown in Tables 7-9. Significant associations with PTC risks were found for rs944289 and rs965513 in females, and for rs1443434 in males. Codominant model was detected as the best model for all the significant comparisons. For rs944289, T allele $(\mathrm{OR}=1.29,95 \% \mathrm{CI}$ : $1.10-1.50, P=0.001)$ and TT homozygote (codominant model: $\mathrm{OR}=1.67,95 \% \mathrm{CI}$ : 1.22-2.28, $P=0.005$ ) significantly increased PTC risk in females, but the risk effect of TT genotype was not significant 
TABLE 4: Association between rs965513 polymorphism and PTC/NG.

\begin{tabular}{|c|c|c|c|c|c|c|}
\hline \multirow{2}{*}{ Genotype/allele } & \multicolumn{3}{|c|}{ PTC vs. NC } & \multicolumn{3}{|c|}{ NG vs. NC } \\
\hline & OR $(95 \% \mathrm{CI})$ & $P$ & AIC & OR $(95 \% \mathrm{CI})^{*}$ & $P^{*}$ & AIC \\
\hline \multicolumn{7}{|l|}{ Codominant } \\
\hline $\mathrm{G} / \mathrm{G}$ & 1.00 & 0.008 & 2362.4 & 1.00 & 0.009 & 1795.4 \\
\hline $\mathrm{G} / \mathrm{A}$ & $1.25(0.99-1.59)$ & & & $0.82(0.60-1.10)$ & & \\
\hline $\mathrm{A} / \mathrm{A}$ & $3.81(1.25-11.63)$ & & & $4.38(1.36-14.11)$ & & \\
\hline \multicolumn{7}{|l|}{ Dominant } \\
\hline $\mathrm{G} / \mathrm{G}$ & 1.00 & 0.021 & 2364.7 & 1.00 & 0.500 & 1802.4 \\
\hline $\mathrm{G} / \mathrm{A}+\mathrm{A} / \mathrm{A}$ & $1.32(1.04-1.67)$ & & & $0.91(0.68-1.21)$ & & \\
\hline \multicolumn{7}{|l|}{ Recessive } \\
\hline $\mathrm{G} / \mathrm{G}+\mathrm{G} / \mathrm{A}$ & 1.00 & 0.013 & 2363.9 & 1.00 & 0.006 & 1795.2 \\
\hline $\mathrm{A} / \mathrm{A}$ & $3.64(1.19-11.10)$ & & & $4.53(1.41-14.59)$ & & \\
\hline \multicolumn{7}{|l|}{ Overdominant } \\
\hline $\mathrm{G} / \mathrm{G}+\mathrm{A} / \mathrm{A}$ & 1.00 & 0.083 & 2367.1 & 1.00 & 0.140 & 1800.6 \\
\hline $\mathrm{G} / \mathrm{A}$ & $1.23(0.97-1.57)$ & & & $0.80(0.59-1.08)$ & & \\
\hline \multicolumn{7}{|l|}{ Allele } \\
\hline G & 1.00 & 0.007 & - & 1.00 & 0.932 & - \\
\hline A & $1.34(1.08-1.67)$ & & & $1.01(0.78-1.32)$ & & \\
\hline
\end{tabular}

*Adjusted by age. PTC, papillary thyroid carcinoma; NG, nodular goiter; NC, normal control.

TABle 5: Association between rs1443434 polymorphism and PTC/NG.

\begin{tabular}{|c|c|c|c|c|c|c|}
\hline \multirow{2}{*}{ Genotype/allele } & \multicolumn{3}{|c|}{ PTC vs. NC } & \multicolumn{3}{|c|}{ NG vs. NC } \\
\hline & OR (95\% CI) & $P$ & AIC & OR $(95 \% \mathrm{CI})^{*}$ & $P^{*}$ & AIC \\
\hline \multicolumn{7}{|l|}{ Codominant } \\
\hline $\mathrm{T} / \mathrm{T}$ & 1.00 & 0.010 & 2339.3 & 1.00 & 0.370 & 1747.5 \\
\hline $\mathrm{T} / \mathrm{G}$ & $1.26(1.01-1.58)$ & & & $0.90(0.68-1.18)$ & & \\
\hline $\mathrm{G} / \mathrm{G}$ & $2.21(1.15-4.23)$ & & & $0.53(0.18-1.51)$ & & \\
\hline \multicolumn{7}{|l|}{ Dominant } \\
\hline $\mathrm{T} / \mathrm{T}$ & 1.00 & 0.011 & 2340.1 & 1.00 & 0.320 & 1746.5 \\
\hline $\mathrm{T} / \mathrm{G}+\mathrm{G} / \mathrm{G}$ & $1.33(1.07-1.65)$ & & & $0.87(0.67-1.14)$ & & \\
\hline \multicolumn{7}{|l|}{ Recessive } \\
\hline $\mathrm{T} / \mathrm{T}+\mathrm{T} / \mathrm{G}$ & 1.00 & 0.022 & 2341.4 & 1.00 & 0.230 & 1746.1 \\
\hline $\mathrm{G} / \mathrm{G}$ & $2.08(1.09-3.99)$ & & & $0.54(0.19-1.54)$ & & \\
\hline \multicolumn{7}{|l|}{ Overdominant } \\
\hline $\mathrm{T} / \mathrm{T}+\mathrm{G} / \mathrm{G}$ & 1.00 & 0.070 & 2343.3 & 1.00 & 0.490 & 1747.0 \\
\hline $\mathrm{T} / \mathrm{G}$ & $1.23(0.98-1.54)$ & & & $0.91(0.69-1.20)$ & & \\
\hline \multicolumn{7}{|l|}{ Allele } \\
\hline $\mathrm{T}$ & 1.00 & 0.003 & - & 1.00 & 0.230 & - \\
\hline G & $1.33(1.10-1.61)$ & & & $1.16(0.91-1.49)$ & & \\
\hline
\end{tabular}

*Adjusted by age. PTC, papillary thyroid carcinoma; NG, nodular goiter; NC, normal control. Bonferroni correction was performed, and $P<0.003$ was defined as statistical significance. Significant $P$ values after Bonferroni correction in bold.

TABLE 6: Cumulative risk alleles of the three SNPs and association with PTC.

\begin{tabular}{|c|c|c|c|c|c|c|c|c|}
\hline No. & PTC & NG & $\mathrm{NC}$ & $\chi^{2}$ & $P$ & $P_{\text {trend }}$ & OR $(95 \% \mathrm{CI})^{*}$ & $P^{*}$ \\
\hline 0 & $146(18.0)$ & $108(21.5)$ & $178(21.7)$ & 29.881 & 0.001 & $<0.001$ & $1.25(1.13-1.37)$ & $<0.001$ \\
\hline 1 & $298(36.8)$ & $194(38.6)$ & $338(41.2)$ & & & & & \\
\hline 2 & $231(28.5)$ & $147(29.3)$ & $222(27.1)$ & & & & & \\
\hline 3 & $111(13.7)$ & $44(8.8)$ & $76(9.3)$ & & & & & \\
\hline 4 & $17(2.1)$ & $8(1.6)$ & $6(0.7)$ & & & & & \\
\hline 5 & $7(0.9)$ & $1(0.2)$ & $0(0.0)$ & & & & & \\
\hline Total & $810(100.0)$ & $502(100.0)$ & $820(100.0)$ & & & & & \\
\hline
\end{tabular}

${ }^{*}$ OR $(95 \% C I)$ and $P$ value were calculated by binary logistic regression for PTC vs. NC. $P_{\text {trend }} P$ value of linear-by-linear association test. PTC, papillary thyroid carcinoma; NG, nodular goiter; NC, normal control. Significant $P$ values in bold.

after Bonferroni correction. For rs965513, A allele $(\mathrm{OR}=1.30$, 95\% CI: $1.01-1.67, P=0.040$ ) and AA homozygote (codominant model: $\mathrm{OR}=4.37,95 \%$ CI: $0.92-20.65, P=0.044)$ significantly increased PTC risk in females, but the associations were not significant after Bonferroni correction any more. No significant association was found in males for 
TABle 7: Association between rs 944289 polymorphism and PTC by gender.

\begin{tabular}{|c|c|c|c|c|c|c|}
\hline \multirow{2}{*}{ Genotype/allele } & \multicolumn{3}{|c|}{ Female } & \multicolumn{3}{|c|}{ Male } \\
\hline & OR (95\% CI) & $P$ & AIC & OR $(95 \% \mathrm{CI})$ & $P$ & AIC \\
\hline \multicolumn{7}{|l|}{ Codominant } \\
\hline $\mathrm{C} / \mathrm{C}$ & 1.00 & 0.005 & 1822.2 & 1.00 & 0.660 & 592.8 \\
\hline $\mathrm{C} / \mathrm{T}$ & $1.22(0.95-1.57)$ & & & $1.22(0.80-1.86)$ & & \\
\hline $\mathrm{T} / \mathrm{T}$ & $1.67(1.22-2.28)$ & & & $1.09(0.62-1.92)$ & & \\
\hline \multicolumn{7}{|l|}{ Dominant } \\
\hline $\mathrm{C} / \mathrm{C}$ & 1.00 & 0.014 & 1824.7 & 1.00 & 0.410 & 591.0 \\
\hline $\mathrm{C} / \mathrm{T}+\mathrm{T} / \mathrm{T}$ & $1.34(1.06-1.69)$ & & & $1.18(0.79-1.76)$ & & \\
\hline \multicolumn{7}{|l|}{ Recessive } \\
\hline $\mathrm{C} / \mathrm{C}+\mathrm{C} / \mathrm{T}$ & 1.00 & 0.005 & 1822.8 & 1.00 & 0.930 & 591.6 \\
\hline $\mathrm{T} / \mathrm{T}$ & $1.48(1.12-1.94)$ & & & $0.98(0.59-1.63)$ & & \\
\hline \multicolumn{7}{|l|}{ Overdominant } \\
\hline $\mathrm{C} / \mathrm{C}+\mathrm{T} / \mathrm{T}$ & 1.00 & 0.970 & 1830.7 & 1.00 & 0.390 & 590.9 \\
\hline $\mathrm{C} / \mathrm{T}$ & $1.00(0.81-1.25)$ & & & $1.18(0.81-1.73)$ & & \\
\hline \multicolumn{7}{|l|}{ Allele } \\
\hline $\mathrm{C}$ & 1.00 & 0.001 & - & 1.00 & 0.600 & - \\
\hline $\mathrm{T}$ & $1.29(1.10-1.50)$ & & & $1.08(0.82-1.42)$ & & \\
\hline
\end{tabular}

Bonferroni correction was performed, and $P<0.003$ was defined as statistical significance. Significant $P$ values after Bonferroni correction in bold.

TABle 8: Association between rs 965513 polymorphism and PTC by gender.

\begin{tabular}{|c|c|c|c|c|c|c|}
\hline \multirow{2}{*}{ Genotype/allele } & \multicolumn{3}{|c|}{ Female } & \multicolumn{3}{|c|}{ Male } \\
\hline & OR $(95 \% \mathrm{CI})$ & $P$ & AIC & OR $(95 \% \mathrm{CI})$ & $P$ & AIC \\
\hline \multicolumn{7}{|l|}{ Codominant } \\
\hline $\mathrm{G} / \mathrm{G}$ & 1.00 & 0.044 & 1787.6 & 1.00 & 0.170 & 580.6 \\
\hline G/A & $1.23(0.93-1.61)$ & & & $1.34(0.82-2.19)$ & & \\
\hline $\mathrm{A} / \mathrm{A}$ & $4.37(0.92-20.65)$ & & & $3.23(0.64-16.23)$ & & \\
\hline \multicolumn{7}{|l|}{ Dominant } \\
\hline $\mathrm{G} / \mathrm{G}$ & 1.00 & 0.070 & 1788.7 & 1.00 & 0.130 & 579.7 \\
\hline $\mathrm{G} / \mathrm{A}+\mathrm{A} / \mathrm{A}$ & $1.28(0.98-1.67)$ & & & $1.44(0.90-2.32)$ & & \\
\hline \multicolumn{7}{|l|}{ Recessive } \\
\hline $\mathrm{G} / \mathrm{G}+\mathrm{G} / \mathrm{A}$ & 1.00 & 0.040 & 1787.8 & 1.00 & 0.150 & 580.0 \\
\hline $\mathrm{A} / \mathrm{A}$ & $4.19(0.89-19.80)$ & & & $3.04(0.61-15.26)$ & & \\
\hline \multicolumn{7}{|l|}{ Overdominant } \\
\hline $\mathrm{G} / \mathrm{G}+\mathrm{A} / \mathrm{A}$ & 1.00 & 0.170 & 1790.0 & 1.00 & 0.280 & 580.9 \\
\hline $\mathrm{G} / \mathrm{A}$ & $1.21(0.92-1.59)$ & & & $1.31(0.80-2.13)$ & & \\
\hline \multicolumn{7}{|l|}{ Allele } \\
\hline G & 1.00 & 0.040 & - & 1.00 & 0.077 & - \\
\hline A & $1.30(1.01-1.67)$ & & & $1.49(0.97-2.28)$ & & \\
\hline
\end{tabular}

TABLE 9: Association between rs1443434 polymorphism and PTC by gender.

\begin{tabular}{|c|c|c|c|c|c|c|}
\hline \multirow{2}{*}{ Genotype/allele } & \multicolumn{3}{|c|}{ Female } & \multicolumn{3}{|c|}{ Male } \\
\hline & OR $(95 \% \mathrm{CI})$ & $P$ & AIC & OR $(95 \% \mathrm{CI})$ & $P$ & AIC \\
\hline \multicolumn{7}{|l|}{ Codominant } \\
\hline $\mathrm{T} / \mathrm{T}$ & 1.00 & 0.170 & 1774.1 & 1.00 & 0.015 & 568.4 \\
\hline $\mathrm{T} / \mathrm{G}$ & $1.17(0.90-1.51)$ & & & $1.60(1.01-2.53)$ & & \\
\hline $\mathrm{G} / \mathrm{G}$ & $1.78(0.86-3.68)$ & & & $4.91(1.03-23.51)$ & & \\
\hline \multicolumn{7}{|l|}{ Dominant } \\
\hline $\mathrm{T} / \mathrm{T}$ & 1.00 & 0.130 & 1773.5 & 1.00 & 0.013 & 568.5 \\
\hline $\mathrm{T} / \mathrm{G}+\mathrm{G} / \mathrm{G}$ & $1.21(0.95-1.56)$ & & & $1.75(1.12-2.72)$ & & \\
\hline \multicolumn{7}{|l|}{ Recessive } \\
\hline $\mathrm{T} / \mathrm{T}+\mathrm{T} / \mathrm{G}$ & 1.00 & 0.140 & 1773.3 & 1.00 & 0.038 & 570.5 \\
\hline $\mathrm{G} / \mathrm{G}$ & $1.71(0.83-3.53)$ & & & $4.37(0.92-20.84)$ & & \\
\hline \multicolumn{7}{|l|}{ Overdominant } \\
\hline $\mathrm{T} / \mathrm{T}+\mathrm{G} / \mathrm{G}$ & 1.00 & 0.300 & 1774.5 & 1.00 & 0.067 & 571.4 \\
\hline $\mathrm{T} / \mathrm{G}$ & $1.15(0.89-1.48)$ & & & $1.53(0.97-2.41)$ & & \\
\hline \multicolumn{7}{|l|}{ Allele } \\
\hline $\mathrm{T}$ & 1.00 & 0.074 & - & 1.00 & 0.005 & - \\
\hline $\mathrm{G}$ & $1.22(0.98-1.52)$ & & & $1.74(1.17-2.59)$ & & \\
\hline
\end{tabular}


rs 965513 and rs944289. For rs1443434, $\mathrm{G}$ allele $(\mathrm{OR}=1.74$, 95\% CI: $1.17-2.59, P=0.005$ ) and GG homozygote (codominant model: $\mathrm{OR}=4.91,95 \% \mathrm{CI}: 1.03-23.51, P=0.015$ ) significantly increased PTC risk in males; however, the associations were not significant after Bonferroni correction. No significant association was found in females for rs 1443434 .

\section{Discussion}

In our present study, we evaluated the effect of TITF1 rs944289 and TITF2 rs965513 and rs1443434 polymorphisms on both PTC and NG, and we found that polymorphisms of rs 944289 and rs1443434 are associated with PTC and NG, and PTC risk increases with the number of risk alleles of the three SNPs. After stratified by gender, a significant association between rs 944289 polymorphism and PTC risk was only observed in females. Moreover, haplotypes T-G-G and T-G-T (rs944289-rs965513-rs1443434) are risk haplotypes of PTC and NG, respectively.

The polymorphism rs944289 predisposes to PTC through PTC susceptibility candidate 3 (PTCSC3) gene. PTCSC3 is a large intergenic noncoding RNA gene [25]. The risk allele of SNP rs944289 suppresses PTCSC3 by destroying a transcription factor-binding site in the promoter of PTCSC3 [26]. Our results showed that rs944289 polymorphism was associated with PTC risk, but no similar result was obtained when we used 1000 genome CHBs as controls, and we speculated that sample size was the main reason to the difference. In 1000 genome online database, only $45 \mathrm{CHBs}$ was genotyped for the three SNPs, whereas in our own case-control study, 896 Han Chinese individuals were included. Moreover, geographical difference between 1000 genome CHBs (Beijing) and our controls (mainly from the Northeast China) may influence on these results. After stratified by gender, a significant association between rs944289 polymorphism and PTC risk was only observed in females, validating that the sex difference is involved in PTC pathogenesis. However, no statistically significant association between rs 944289 and the risk of female breast cancer was also observed in a Chinese population [27], suggesting that rs 944289 polymorphism may be required for female PTC specially, rather than breast cancer.

Although several meta-analyses have been performed to explore the association between these SNPs and thyroid cancer susceptibility, they still get no consistent result. Study of Chen et al. revealed the rs965513 polymorphism is significantly associated with risk of differentiated thyroid carcinoma (DTC) in Caucasians, but not in East Asians. The rs944289 polymorphism is associated with DTC in both Caucasians and East Asians [28]. Kang et al.'s study showed that the polymorphisms of rs944289 and rs965513 are not associated with thyroid cancer risk, but the rs1443434 polymorphism was significantly associated with thyroid cancer risk in overall population [29]. In Zhuang et al.'s study, rs965513 A allele is significantly associated with increased risk of thyroid cancer in both Caucasians and Asians [30]. In Gao et al.'s study, significant associations are found between the polymorphisms of rs944289 and rs965513 and risk of PTC risk in Caucasians and Asians [31]. In Figlioli et al.'s study, using GWAS and meta-analysis approaches confirms rs965513 as a risk locus of DTC [32].

Our present study focused on associations of the three SNPs (rs944289, rs965513, and rs1443434) and PTC risk in northern Chinese Han populations, whose ethnic background is different from the previous studies [16-18]. As a hospital-based case-control designed study, we must admit the potential selection bias which is common in such studies. In addition, sample size of our study (861 PTCs, 562 NGs, and $896 \mathrm{NCs}$ ) is relatively small, influencing the statistical power. Besides, environmental factors, such as smoking and drinking, are also remarkable risk factors for thyroid cancer, which were not analyzed in our study. Furthermore, we only selected three SNPs of interest for analysis, not taking other molecular subtyping into account. $\mathrm{BRAF}^{\mathrm{V} 600 \mathrm{E}}$ mutation, for example, which is an important mutation in thyroid cancer, may influence the genetic structure of the studied population, affecting the risk evaluation. Finally, studies on mechanism between rs944289 and rs1443434 polymorphisms and PTC should be performed to demonstrate the effect. Nevertheless, our study provides possible biomarkers for PTC diagnose and provides clews for further mechanism study, especially in northern Chinese Han populations.

\section{Conclusions}

The results of this study showed that polymorphisms of rs944289 and rs1443434 are associated with both PTC and NG in Chinese Han populations, and PTC risk increases with the number of total risk alleles of the three SNPs. Moreover, haplotypes T-G-G and T-G-T (rs944289rs965513-rs1443434) are risk haplotypes of PTC and NG, respectively.

\section{Data Availability}

The data used to support the findings of this study are available from the corresponding author upon request.

\section{Conflicts of Interest}

The authors declare that they have no conflicts of interest.

\section{Authors' Contributions}

Xin Zhang and Yulu Gu contributed equally to this work.

\section{Acknowledgments}

The authors would like to thank Lizhe Ai, Guang Yang, Qingqing, Zhang, Hongqin $\mathrm{Xu}$, Chong Wang, and Wen Wen for their assistance in data collection and experiment. This work was supported by the National Natural Science Foundation of China (grant numbers 81602907, 81903389, and 81702606), the funds from Science and Technology Commission of Jilin Province (grant number 17240BGH010135414), and the funds from Commission of Health and Family Planning of Jilin Province (grant number 2017Q037). The National Natural Science Foundation of China plays role in the study design and in the manuscript 
writing, editing, approval, and decision to publish, and the others play role in sample collection, analysis, and interpretation of data.

\section{Supplementary Materials}

Table S1: the PCR amplification primer sequence. Table S2: clinical characteristics of PTCs $(n=861)$. Table S3: linkage disequilibrium between the three SNPs. Table S4: genotype and allele distributions of the three SNPs in PTCs, NGs, and 1000genome CHBs. Table S5: summary of Quanto results for power calculation. Table S6: association between rs944289 polymorphism and PTC/NG with 1000genome $\mathrm{CHB}$ as controls. Table S7: association between rs965513 polymorphism and PTC/NG with 1000genome CHB as controls. Table S8: association between rs1443434 polymorphism and PTC/NG with 1000genome CHB as controls. Table S9: associations between TITF1/TITF2 haplotypes and risk of PTC. Table S10: associations between TITF1/TITF2 haplotypes and risk of NG. Table S11: genotype and allele frequencies of the three SNPs by gender ( $n, \%)$. (Supplementary Materials)

\section{References}

[1] R. L. Siegel, K. D. Miller, and A. Jemal, "Cancer statistics, 2018," CA: A Cancer Journal for Clinicians, vol. 68, no. 1, pp. 7-30, 2018.

[2] L. G. T. Morris, R. M. Tuttle, and L. Davies, "Changing trends in the incidence of thyroid cancer in the United States," JAMA Otolaryngology-Head \& Neck Surgery, vol. 142, no. 7, pp. 709-711, 2016.

[3] Y. Hwangbo and Y. J. Park, "Genome-wide association studies of autoimmune thyroid diseases, thyroid function, and thyroid cancer," Endocrinology and Metabolism, vol. 33, no. 2, pp. 175-184, 2018.

[4] K. Czene, P. Lichtenstein, and K. Hemminki, "Environmental and heritable causes of cancer among 9.6 million individuals in the Swedish family-cancer database," International Journal of Cancer, vol. 99, no. 2, pp. 260-266, 2002.

[5] M. Fallah, E. Pukkala, L. Tryggvadottir et al., "Risk of thyroid cancer in first-degree relatives of patients with non-medullary thyroid cancer by histology type and age at diagnosis: a joint study from five Nordic countries," Journal of Medical Genetics, vol. 50, no. 6, pp. 373-382, 2013.

[6] Y. K. Lee, D. Y. Shin, H. Shin, and E. J. Lee, "Sex-specific genetic influence on thyroid-stimulating hormone and free thyroxine levels, and interactions between measurements: KNHANES 2013-2015," PLoS One, vol. 13, no. 11, Article ID e0207446, 2018.

[7] A. D. Tarnoki, D. L. Tarnoki, G. Speer et al., "Genetic and environmental influence on thyroid gland volume and thickness of thyroid isthmus: a twin study," Archives of Endocrinology and Metabolism, vol. 59, no. 6, pp. 487-494, 2015.

[8] M. Xing, "Molecular pathogenesis and mechanisms of thyroid cancer," Nature Reviews Cancer, vol. 13, no. 3, pp. 184-199, 2013.

[9] J. Xie, Y. Fan, and X. Zhang, "Molecular mechanisms in differentiated thyroid cancer," Frontiers in Bioscience, vol. 21, no. 1, pp. 119-129, 2016.
[10] S. Guo, Y.-L. Wang, Y. Li et al., "Significant SNPs have limited prediction ability for thyroid cancer," Cancer Medicine, vol. 3, no. 3, pp. 731-735, 2014.

[11] J. Gudmundsson, G. Thorleifsson, J. K. Sigurdsson et al., “A genome-wide association study yields five novel thyroid cancer risk loci," Nature Communications, vol. 8, p. 14517, 2017.

[12] P. Geng, J. Ou, J. Li et al., "TITF1 and TITF2 loci variants indicate significant associations with thyroid cancer," Endocrine, vol. 50, no. 3, pp. 598-607, 2015.

[13] A. Bychkov, V. Saenko, M. Nakashima et al., "Patterns of FOXE1 expression in papillary thyroid carcinoma by immunohistochemistry," Thyroid, vol. 23, no. 7, pp. 817-828, 2013.

[14] P. Lapi, P. E. Macchia, L. Chiovato et al., "Mutations in the gene encoding thyroid transcription factor-1 (TTF-1) are not a frequent cause of congenital hypothyroidism $(\mathrm{CH})$ with thyroid dysgenesis," Thyroid, vol. 7, no. 3, pp. 383-387, 1997.

[15] N. Dathan, R. Parlato, A. Rosica, M. De Felice, and R. Di Lauro, "Distribution of thetitf2/foxe1 gene product is consistent with an important role in the development of foregut endoderm, palate, and hair," Developmental Dynamics, vol. 224, no. 4, pp. 450-456, 2002.

[16] M. Matsuse, M. Takahashi, N. Mitsutake et al., "The FOXE1 and NKX2-1 loci are associated with susceptibility to papillary thyroid carcinoma in the Japanese population," Journal of Medical Genetics, vol. 48, no. 9, pp. 645-648, 2011.

[17] C. M. Pereda, F. Lesueur, M. Pertesi et al., "Common variants at the 9q22.33, 14q13.3 and ATM loci, and risk of differentiated thyroid cancer in the Cuban population," $B M C \mathrm{Ge}$ netics, vol. 16, no. 1, 2015.

[18] J. Gudmundsson, P. Sulem, D. F. Gudbjartsson et al., "Common variants on 9q22.33 and 14q13.3 predispose to thyroid cancer in European populations," Nature Genetics, vol. 41, no. 4, pp. 460-464, 2009.

[19] D. S. Cooper, G. M. Doherty, B. R. Haugen et al., "Revised American Thyroid Association Management Guidelines for patients with thyroid nodules and differentiated thyroid cancer," Thyroid, vol. 19, no. 11, pp. 1167-1214, 2009.

[20] E. Bonora, C. Rizzato, C. Diquigiovanni et al., "TheFOXE1locus is a major genetic determinant for familial nonmedullary thyroid carcinoma," International Journal of Cancer, vol. 134, no. 9, pp. 2098-2107, 2014.

[21] Y.-L. Wang, S.-H. Feng, S.-C. Guo et al., "Confirmation of papillary thyroid cancer susceptibility loci identified by genome-wide association studies of chromosomes 14q13, 9q22, $2 \mathrm{q} 35$ and $8 \mathrm{p} 12$ in a Chinese population," Journal of Medical Genetics, vol. 50, no. 10, pp. 689-695, 2013.

[22] M. Takahashi, V. A. Saenko, T. I. Rogounovitch et al., "The FOXE1 locus is a major genetic determinant for radiationrelated thyroid carcinoma in Chernobyl," Human Molecular Genetics, vol. 19, no. 12, pp. 2516-2523, 2010.

[23] Y. Gu, X. Liu, Y. Yu et al., "Association of ATM gene polymorphism with PTC metastasis in female patients," International Journal of Endocrinology, vol. 2014, Article ID 370825, 7 pages, 2014.

[24] X. Sole, E. Guino, J. Valls, R. Iniesta, and V. Moreno, "SNPStats: a web tool for the analysis of association studies," Bioinformatics, vol. 22, no. 15, pp. 1928-1929, 2006.

[25] J. Jendrzejewski, H. He, H. S. Radomska et al., "The polymorphism rs944289 predisposes to papillary thyroid carcinoma through a large intergenic noncoding RNA gene of tumor suppressor type," Proceedings of the National Academy of Sciences, vol. 109, no. 22, pp. 8646-8651, 2012. 
[26] J. Jendrzejewski, A. Thomas, S. Liyanarachchi et al., "PTCSC3 is involved in papillary thyroid carcinoma development by modulating S100A4 gene expression," The Journal of Clinical Endocrinology \& Metabolism, vol. 100, no. 10, pp. E1370E1377, 2015.

[27] T. Xu, X.-X. Hu, X.-X. Liu et al., "Association between SNPs in long non-coding RNAs and the risk of female breast cancer in a Chinese population," Journal of Cancer, vol. 8, no. 7, pp. 1162-1169, 2017.

[28] Y. H. Chen and Y. Q. Zhang, "Exploration of the association between FOXE1 gene polymorphism and differentiated thyroid cancer: a meta-analysis," BMC Medical Genetics, vol. 19, no. 1, p. 83, 2018.

[29] J. Kang, X.-Z. Deng, Y.-B. Fan, and B. Wu, "Relationships of FOXE1 and ATM genetic polymorphisms with papillary thyroid carcinoma risk: a meta-analysis," Tumor Biology, vol. 35, no. 7, pp. 7085-7096, 2014.

[30] Y. Zhuang, W. Wu, H. Liu, and W. Shen, "Common genetic variants on FOXE1 contributes to thyroid cancer susceptibility: evidence based on 16 studies," Tumor Biology, vol. 35, no. 6, pp. 6159-6166, 2014.

[31] Y. Gao, F. Chen, S. Niu, S. Lin, and S. Li, "Replication and meta-analysis of common gene mutations in TTF1 and TTF2 with papillary thyroid cancer," Medicine, vol. 94, no. 36, p. e1246, 2015

[32] G. Figlioli, R. Elisei, C. Romei et al., "A comprehensive metaanalysis of case-control association studies to evaluate polymorphisms associated with the risk of differentiated thyroid carcinoma," Cancer Epidemiology Biomarkers \& Prevention, vol. 25 , no. 4, pp. 700-713, 2016. 